\title{
Use of idarucizumab in reversing dabigatran anticoagulant effect: a critical appraisal
}

This article was published in the following Dove Press journal:

Therapeutics and Clinical Risk Management

\author{
Marco Proietti ${ }^{1-3}$ \\ Giuseppe Boriani ${ }^{4}$ \\ 'Department of Neuroscience, IRCCS - \\ Istituto di Ricerche Farmacologiche \\ "Mario Negri", Milan, Italy; ${ }^{2}$ Institute \\ of Cardiovascular Sciences, University \\ of Birmingham, Birmingham, UK; \\ ${ }^{3}$ Department of Internal Medicine \\ and Medical Specialties, Sapienza- \\ University of Rome, Rome, Italy; \\ ${ }^{4}$ Department of Biomedical, Metabolic \\ and Neural Sciences, University of \\ Modena and Reggio Emilia, Policlinico \\ di Modena, Modena, Italy
}

\begin{abstract}
Use of non-vitamin $\mathrm{K}$ antagonist oral anticoagulants is spreading in the real world. Despite that, a strong need for antidotes/reversal agents is still reported by several physicians. Idarucizumab is a humanized monoclonal antibody fragment that binds specifically to dabigatran. Idarucizumab was approved in 2015 by the US Food and Drugs Administration and European Medicines Agency for reversal of anticoagulation activity in dabigatran-treated patients. This review briefly summarizes the experimental evidence about effectiveness and safety of idarucizumab. Furthermore, we review the current recommendations and experts' point of view about the use of antidotes/reversal agents in patients reporting a major bleeding event.
\end{abstract}

Keywords: anticoagulant drugs, non-vitamin K antagonist oral anticoagulants, reversal agents, idarucizumab, major bleeding

\section{Introduction}

In 2009 the introduction of non-vitamin K antagonist oral anticoagulants (NOACs) in the market as an effective and safer alternative for the prevention of thromboembolic events in atrial fibrillation (AF) patients ${ }^{1}$ and the treatment and prevention of recurrences in patients with venous thromboembolic events (VTE) ${ }^{2}$ drastically changed the current pharmacopeia and routine clinical treatment of these two conditions. Dabigatran etexilate (aDabiFab) is the only direct thrombin inhibitor among the authorized NOACs and was the first one to be demonstrated more effective and safer than warfarin for $\mathrm{AF}^{3}$ and has a similar efficacy and safety for VTE patients. ${ }^{4}$

Despite several practical aspects that favor NOACs over warfarin (no need of continuous laboratory monitoring, limited drug-drug interactions, no food interactions, fixed-dose regimen, predictable anticoagulant activity, etc.), the absence of specific antidote and reversal agents has been a significant caveat to widespread use of these drugs. ${ }^{5,6}$ In order to face this issue, in the last years several companies started developing antidotes/reversal agents for NOACs. ${ }^{6}$ Among them, idarucizumab is a specific reversal agent for dabigatran and is also the first one to receive approval from the US Food and Drugs Administration (FDA) and European Medicines Agency in 2015 (Table 1).

Idarucizumab was tested in two different randomized clinical trials, ${ }^{7-9}$ which demonstrated it to be an effective and safe reversal agent. This narrative review aims to describe the main characteristics of idarucizumab and summarize the most relevant evidence about its use in patients receiving dabigatran for thromboprophylaxis in those affected with AF and VTE. 
Table I Main characteristics of idarucizumab

\begin{tabular}{|c|c|}
\hline Feature & Idarucizumab \\
\hline Structure & Humanized Fab fragment \\
\hline \multirow[t]{2}{*}{ Molecular mechanism } & Noncompetitive binding \\
\hline & (350-fold higher affinity than thrombin) \\
\hline \multirow[t]{2}{*}{ Route of delivery } & Two $50-\mathrm{mL}$ intravenous bolus infusions \\
\hline & 15 minutes apart, equal to $5 \mathrm{~g}$ of drug \\
\hline Regulatory aspects & Approved by FDA and EMA \\
\hline Latency & Immediate onset of reversal \\
\hline Duration of effect & 12-24 hours \\
\hline Studies & NCTOI 688830,7 NCT02 I 04947 (REVERSE-AD) ${ }^{8,9}$ \\
\hline Costs & $\$ 3,662.06$ (US) - €3,909.9I (EU) per unit \\
\hline
\end{tabular}

Abbreviations: EMA, European Medicines Agency; FDA, Food and Drug Administration.

\section{Mechanism of action}

Idarucizumab is a humanized monoclonal antibody fragment specifically and potently inhibiting aDabiFab. It neutralizes dabigatran activity in a 1:1 stoichiometric relation in both in vitro and in vivo contexts (Figure 1; Table 1). ${ }^{10}$

The original molecule has been produced from dabigatranhapten immunized mice. After that, the antibodies were humanized removing the variable regions from both heavy and light mouse antibodies' chains and hybridized with human IgG constant regions. The variable region of the Fab has been humanized through a design and screening process. $^{10}$

In the original study, the parental mouse and humanized Fab antibodies showed similar in vitro inhibitory activity and clotting times, after the presence of dabigatran was reduced to baseline at antibody concentrations $>10 \mathrm{nM}$, indicating complete neutralization of dabigatran at equimolar concentrations of dabigatran and aDabiFab. ${ }^{10}$

The inhibition activity is due to the noncompetitive binding of dabigatran to a concave region present in the interface of the aDabiFab variable domain. Hydrophobic interactions, $\mathrm{H}$-bonds, and a salt bridge mediate the binding. Idarucizumab binds dabigatran with a 350-fold higher affinity than thrombin (Table 1). ${ }^{10}$ In vivo experiments confirmed in vitro results, with a complete reversal of anticoagulant activity within 1 minute of injection in the mice, sustained during the subsequent 25 minutes, despite the continuous infusion of further dabigatran. ${ }^{10}$

Despite the similarities between aDabiFab and thrombin in recognition and binding of dabigatran, aDabiFab did not show any thrombin-like activity, and in the absence of dabigatran, in vitro studies did not show any effect of the compound on the coagulation pathways. ${ }^{10}$

\section{Experimental evidence}

So far, idarucizumab has been tested in two studies. ${ }^{7-9}$ The first one was a randomized, placebo-controlled, doubleblind Phase I trial. In this study, 47 healthy male volunteers aged 18-45 years with normal body mass index and no relevant health conditions were enrolled to be allocated in four idarucizumab sequential dose groups ( $1 \mathrm{~g}, 2 \mathrm{~g}$ and $4 \mathrm{~g}$ [as one 5 minutes intravenous infusion] and $5 \mathrm{~g}$ plus $2.5 \mathrm{~g}$ [as two 5 minutes intravenous infusions 1 hour apart]) and randomized to receive idarucizumab or placebo in a $3: 1$ ratio. All the subjects received dabigatran $110 \mathrm{mg}$ twice

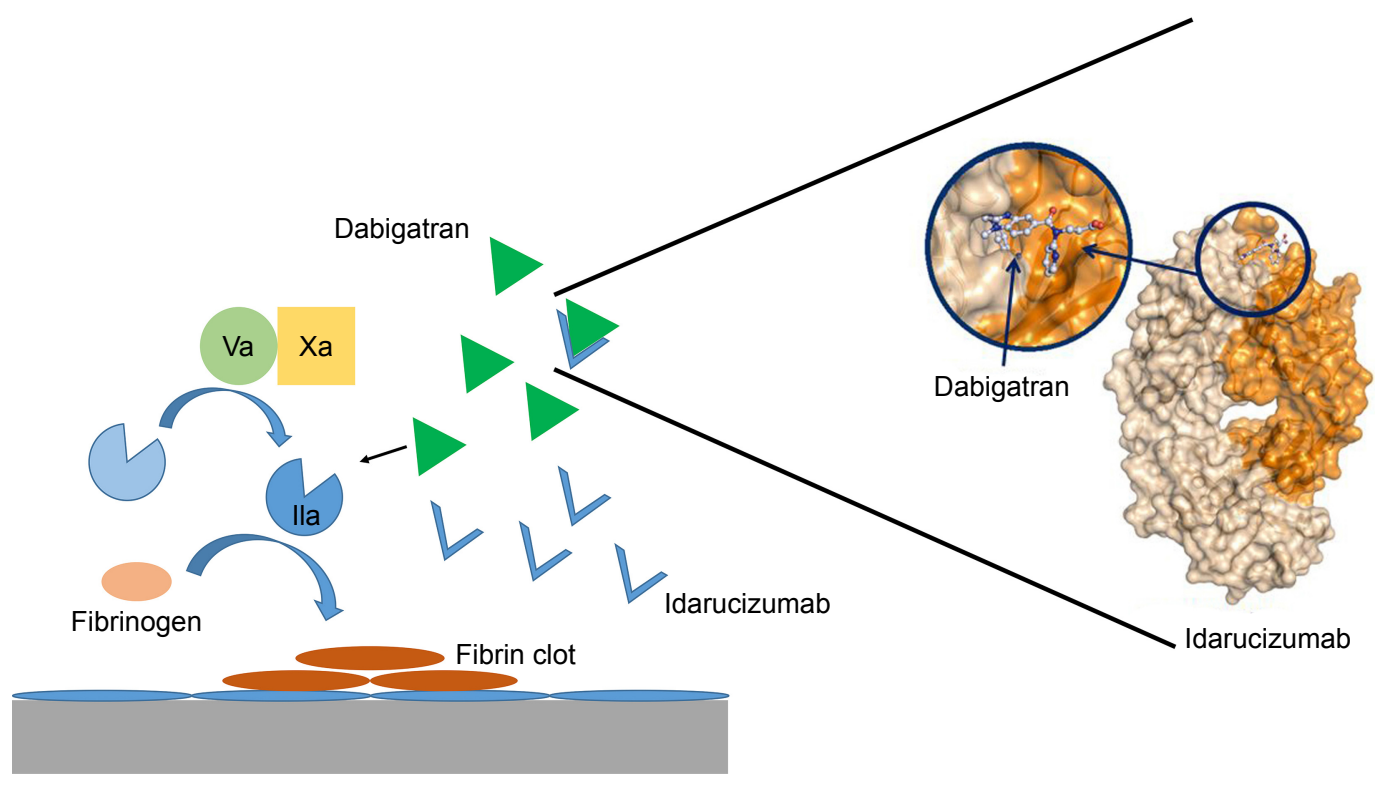

Figure I Idarucizumab and dabigatran in the context of the hemostatic process. 
daily for 3 days. At day 4, after the last dabigatran dose, the subjects allocated in each group received the randomized treatment. ${ }^{7}$

After receiving dabigatran, all the subjects showed a consistent increase in all coagulation tests examined (diluted thrombin time [dTT], ecarin clotting time [ECT], thrombin time, activated partial thromboplastin time [aPTT], activated clotted time). All idarucizumab doses showed an immediate and complete reversal of the dabigatran-induced increase in dTT, which was reduced to less than the upper limit of normal and extremely close to the baseline values. ${ }^{7}$ The reduction in dTT was consistent for all the idarucizumab doses, except for $1 \mathrm{~g}$ which showed a new increase in dTT after finishing the infusion. Similar evidence was obtained for the other tested essays. ${ }^{7}$ A dose-dependent effect was found when examining the ratio reduction in the four doses tested. Indeed, $1 \mathrm{~g}$ idarucizumab infusion resulted in a $74 \%$ dTT reduction compared with placebo infusion, $2 \mathrm{~g}$ in a $94 \%$ reduction and so on progressively up to $99 \%$ reduction with $5 \mathrm{~g}$ plus $2.5 \mathrm{~g}$. Furthermore, the infusion of idarucizumab showed normalization of other coagulation indexes, and completely canceled any effect of dabigatran. ${ }^{7}$

The main Phase III study to test efficacy and safety of idarucizumab in reversing dabigatran anticoagulant effect has been the Reversal Effects of Idarucizumab on Active Dabigatran (RE-VERSE AD). ${ }^{8,911}$ Results of the RE-VERSE AD were firstly published in 2015 on a smaller cohort while the study was still ongoing, ${ }^{8}$ which led to FDA approval, while full results of the study, on the entire cohort, were published in $2017 .{ }^{9}$ We reported data mostly based on the full cohort report.

In this multicenter, prospective, single cohort study, 503 patients receiving dabigatran were enrolled to receive $5 \mathrm{~g}$ of intravenous idarucizumab in two distinct boluses, no more than 15 minutes apart. The primary efficacy end point was the maximum percentage of reversal of the anticoagulation effect of dabigatran up until 4 hours after the second idarucizumab infusion. ${ }^{9}$ After the infusion, dTT, ECT, and aPTT were sequentially tested to evaluate the reversal effect. Also, the concentration of unbound dabigatran was tested. All the patients enrolled were divided into two groups: all the patients presenting with an uncontrollable or life-threatening major bleeding were enrolled in group A and all those needing surgery or another invasive procedure that could not be delayed and therefore requiring complete and normal hemostasis were enrolled in group B.

Both the groups were substantially similar regarding the baseline characteristics, dTT, and ECT, which were prolonged in more than $90 \%$ of patients (461 out of 503) at study entry, went back to normal values immediately after the first infusion, with a percentage of reversal within the 4 hours from infusion equal to $100 \%$. The reversal was sustained up to 24 hours. ${ }^{9}$ The percentage of unbound dabigatran remained consistently $<20 \mathrm{ng}$ for 24 hours in most of the patients. In $23 \%$ of patients, a reappearance of dabigatran levels $>20 \mathrm{ng}$ occurred after 12 hours, with 67 of them having elevated levels at 24 hours. In 10 of these patients, being included in group $\mathrm{A}$, the reappearance of unbound dabigatran was associated with relapse in bleeding. ${ }^{9}$

Among 203 patients in group A, for which the assessment of bleeding cessation could have been performed, 134 $(67.7 \%)$ of them had the bleeding cessation confirmed within 24 hours, with a median $(95 \% \mathrm{CI})$ time to hemostasis of 2.5 hours (2.2-3.9 hours). ${ }^{9}$ Different from the entire cohort, data coming from the initial report showed a longer median time to bleeding cessation (11.4 hours). ${ }^{8}$ For the patients in group B, periprocedural hemostasis was considered as normal in $184(93.4 \%)$ patients, while no patients had severely abnormal hemostasis. ${ }^{9}$

Regarding the safety of idarucizumab infusion, thrombotic events occurred in 4.8\% (24 patients) of the entire cohort of 503 patients. In only three cases, the thrombotic event was fatal. In the follow-up observation, 30- and 90-day mortality rates were similar between the two groups $(13.5 \%$ and $12.6 \%$ for groups $\mathrm{A}$ and $\mathrm{B}$, respectively; $18.8 \%$ and $18.9 \%$ for groups $\mathrm{A}$ and $\mathrm{B}$, respectively). ${ }^{9}$

\section{Clinical usefulness and impact on real world}

Several data report that the magnitude of the fear of a major bleeding event occurrence is fourfold higher than the fear of any thromboembolic event for any physician prescribing oral anticoagulant therapy. ${ }^{12}$ The absence of any possible direct reversal agent has been postulated as a possible caveat to the use of NOACs. ${ }^{5}$ Theoretically speaking, several nonspecific reversal strategies can be used even with NOACs (prothrombin complex concentrate, recombinant FVIIa, etc). ${ }^{6}$ Notwithstanding, to create something that could resemble the role of vitamin $\mathrm{K}$ in reversal of warfarin-related anticoagulation, clinical research started to develop several possible specific reversal agents, even though so far only idarucizumab was authorized for clinical use, while other drugs are still in the premarket phase despite some relevant studies. ${ }^{13,14}$

So far, only scarce and sparse data have been published about its use in daily clinical routine. ${ }^{15}$ Only small case series have been documented with not enough evidence 
to understand to what extent the use of idarucizumab can effectively affect the clinical course of patients presenting with acute bleeding or with a need for immediate reversal for urgent surgery. In a small retrospective review, the authors clearly stated that it remained unclear in which clinical situations idarucizumab would be clinically indicated. ${ }^{15}$

In the recent 2016 European Society of Cardiology (ESC) guidelines on management of AF patients, the management of bleeding events in anticoagulated patients was discussed. ${ }^{16}$ In the occurrence of moderate-severe bleeding in patients taking NOACs, the guidelines firstly recommend to manage patients' conditions in order to stabilize the hemodynamics and treat (if possible) the causes of bleeding, as well as the use of oral charcoal if the assumption of the drugs is quite recent. ${ }^{16}$ Only in the occurrence of severe or life-threatening bleeding, the guidelines recommend the use of specific antidotes/reversal strategies. ${ }^{16}$

These recommendations were derived from the joint position paper of the ESC Working Group on Cardiovascular Pharmacotherapy and the ESC Working Groups on Thrombosis. ${ }^{17}$ In this position paper, a group of experts revised all the available evidence related to the risk of bleeding in patients taking NOACs and about effectiveness and safety of the available NOACs' reversal agents. The experts' consensus established that while the effectiveness of these agents is very likely, in particular for idarucizumab, in effectively reversing the anticoagulant effect of the various NOACs, it is not clear if this reversal can significantly affect the occurrence of adverse clinical events. ${ }^{17}$ Furthermore, it is still unclear whether the sudden termination of anticoagulation in patients with a high thromboembolic risk may imply an increase in the risk of thromboembolic events, ${ }^{17}$ which could then jeopardize the final net clinical benefit. In the case of idarucizumab, we should also take into consideration the very high price of the drug, and in this light future cost-effectiveness evaluation would be needed to understand in what conditions its use is justified concerning cost-effectiveness. All these considerations made the expert consensus deliberate about the exclusive use of reversal agents in specific situations, after a careful consideration of various pharmacological and clinical issues, and only for those patients in whom every other measure had failed. ${ }^{17}$ This attitude is also justified by the relatively low incidence of severe/life-threatening bleeding events with NOACs, ${ }^{18}$ as well as the short half-life of these agents.

A similar approach has been suggested in the "American College of Cardiology Expert Consensus Decision Pathway on Management of Bleeding in Patients on Oral Anticoagulants", in which the use of reversal agents was recommended only when the bleed occurred to a critical site or in the case of life-threatening bleeding. ${ }^{19}$ This document, differently from the other mentioned above, ${ }^{17}$ also supports the use of routine laboratory tests to evaluate the NOAC circulating levels indirectly. Even if some indications can be drawn in patients taking dabigatran, in particular from aPTT levels, normal levels of aPTT cannot exclude on-therapy levels; therefore, the usefulness of routine laboratory test in the evaluation of NOACs patients actively bleeding is limited. ${ }^{19}$

Taking into consideration all the evidence reported, we believe that several considerations could be drawn.

Firstly, based on the experience in the use of vitamin $\mathrm{K}$ to treat patients who received vitamin $\mathrm{K}$ antagonist overdose or who presented with clinically relevant bleeding, it appears that in the past, physicians much likely overestimated the need for some specific antidotes for NOACs. Secondly, the clinical measures to manage and contain major bleeding effects are not considered valuable procedures to treat these patients, in the general physicians' belief. Thirdly, possibly a strong misunderstanding exists between what the words "antidote" and "reversal" mean. According to Collins dictionary (https://www.collinsdictionary.com) "an antidote is a chemical substance that stops or controls the effect of a poison"; conversely, to reverse is defined as "to turn or set in an opposite direction, order, or position" or alternatively "to change into something different or contrary; alter completely". Most likely, based on the reported definitions, the physicians do not merely need an antidote or a reversal agent, since the real need is to provide an effective treatment for stopping a major bleeding event. Indeed, even if an antidote stops the anticoagulant activity, it does not control the active bleeding; conversely, to name a substance as a reversal agent, it should restore the coagulation activity with the risk to result in a procoagulant effect, as the experts underlined. Considering the data coming from the RE-VERSE AD, we can state that idarucizumab consistently inhibits dabigatran anticoagulant activity, but the question of how much this results in a significant benefit on major outcomes, as a consequence of its use in the real world, is still open.

All the evidence point out that a change in physicians' view is needed on the management of actively bleeding anticoagulated patients. Indeed, even though an active substance "blocking" the anticoagulant activity (a blocker, not an antidote or a reversal agent) could represent an important aid for some specific situations, the most important approach in actively bleeding patients is to sustain the patients' conditions and physically "constrain" the bleeding. Furthermore, in 
Box I Reasonable indications for the use of idarucizumab in dabigatran-treated patients

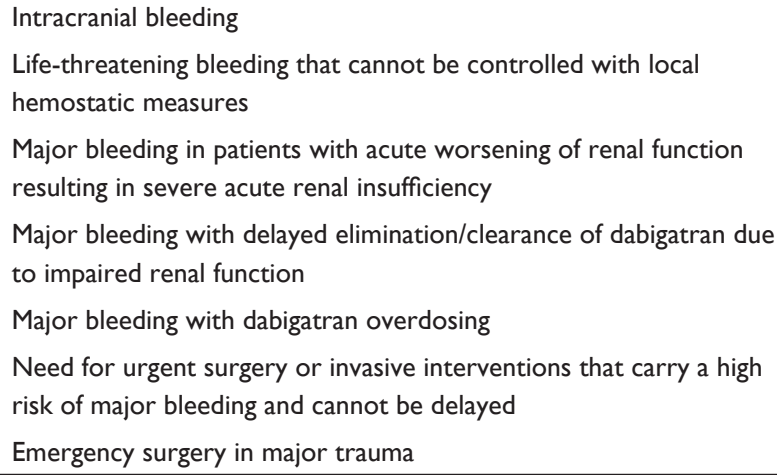

those patients undergoing urgent procedures, while available data from the real world so far seem to report large underuse, ${ }^{15}$ a recent review of NOACs from the surgical point of view underlines how the use of antidotes/reversal agents is limited to very few patients. ${ }^{20}$ This document underlines that the main issue for each surgeon is to precisely measures the patient's ongoing anticoagulant activity, to reassure the operator before to proceed with a surgical procedure, even an urgent one. Given the lack of availability of these tests on a large scale, a valid approach that could be suggested is to delay as much as possible the surgery, waiting long enough to be sure that the pharmacological activity is ceased..$^{20}$ Unfortunately, this approach in some cases could be not feasible or quite risky, and further data are needed to better guide clinical decision-making.

Notwithstanding, keeping in mind all the limitations we underlined above, we think that a number of specific situations can be identified, in which it would be reasonable to consider using idarucizumab to control the anticoagulation activity (Box 1), adhering to the guidelines and expert positions recommending its use exclusively in extremely severe bleeding and critically clinical conditions.

All these aspects, together with the absence of strong data on the clinical effectiveness in reducing major adverse events, as well as the risk of rebound increased thromboembolic risk, support the claim for further stronger evidence clarifying these aspects. ${ }^{21}$

\section{Conclusion}

Idarucizumab has been proved to reverse rapidly, effectively, and consistently anticoagulant activity in dabigatran-treated patients. Despite the strong evidence pointing out the effectiveness and safety of idarucizumab, strong data on patient outcomes are still needed to better target the use of idarucizumab, according to specific high-risk conditions, as well as for other antidotes/reversal agents, in the context of real-world clinical practice.

\section{Disclosure}

MP received consulting fees from Boehringer Ingelheim outside the submitted work; GB received personal fees from Medtronic, Boston, Boehringer and Biotronik, outside the submitted work. The authors report no other conflicts of interest in this work.

\section{References}

1. Ruff CT, Giugliano RP, Braunwald E, et al. Comparison of the efficacy and safety of new oral anticoagulants with warfarin in patients with atrial fibrillation: a meta-analysis of randomised trials. Lancet. 2014; 383(9921):955-962.

2. Gómez-Outes A, Terleira-Fernández AI, Lecumberri R, Suárez-Gea ML, Vargas-Castrillón E. Direct oral anticoagulants in the treatment of acute venous thromboembolism: a systematic review and meta-analysis. Thromb Res. 2014;134(4):774-782.

3. Connolly SJ, Ezekowitz MD, Yusuf S, et al. Dabigatran versus warfarin in patients with atrial fibrillation. $N$ Engl J Med. 2009;361(12): $1139-1151$.

4. Schulman S, Kearon C, Kakkar AK, et al. Dabigatran versus warfarin in the treatment of acute venous thromboembolism. $N$ Engl J Med. 2009;361(24):2342-2352.

5. Schulman S. New oral anticoagulant agents - general features and outcomes in subsets of patients. Thromb Haemost. 2014;111(4):575-582.

6. Proietti M, Lip GYH. Antidotes to non-vitamin K oral anticoagulants: necessary or not? Expert Opin Pharmacother. 2015;16(11):1573-1576.

7. Glund S, Stangier J, Schmohl M, et al. Safety, tolerability, and efficacy of idarucizumab for the reversal of the anticoagulant effect of dabigatran in healthy male volunteers: a randomised, placebo-controlled, doubleblind phase 1 trial. Lancet. 2015;386(9994):680-690.

8. Pollack CV, Reilly PA, Eikelboom J, et al. Idarucizumab for dabigatran reversal. N Engl J Med Overseas Ed. 2015;373(6):511-520.

9. Pollack CV, Reilly PA, van Ryn J, et al. Idarucizumab for dabigatran reversal - full cohort analysis. N Engl J Med. 2017;377(5):431-441.

10. Schiele F, van Ryn J, Canada K, et al. A specific antidote for dabigatran: functional and structural characterization. Blood. 2013;121(18): 3554-3562.

11. Pollack CV, Reilly PA, Bernstein R, et al. Design and rationale for RE-VERSE AD: a phase 3 study of idarucizumab, a specific reversal agent for dabigatran. Thromb Haemost. 2015;114(1):198-205.

12. Raparelli V, Proietti M, Cangemi R, Lip GY, Lane DA, Basili S. Adherence to oral anticoagulant therapy in patients with atrial fibrillation. Focus on non-vitamin $\mathrm{K}$ antagonist oral anticoagulants. Thromb Haemost. 2017;117(2):209-218.

13. Siegal DM, Curnutte JT, Connolly SJ, et al. Andexanet alfa for the reversal of factor Xa inhibitor activity. N Engl J Med. 2015;373(25): 2413-2424.

14. Enriquez A, Lip GY, Baranchuk A. Anticoagulation reversal in the era of the non-vitamin K oral anticoagulants. Europace. 2016;18(7):955-964.

15. Ebinger J, Granger CB, Zhu A, Chang A, Henry TD. Idarucizumab since FDA approval: use in the real-world. Am Heart J. 2017;193:93-94.

16. Kirchhof P, Benussi S, Kotecha D, et al. 2016 ESC Guidelines for the management of atrial fibrillation developed in collaboration with EACTS. Eur Heart J. 2016;37(38):2893-2962.

17. Niessner A, Tamargo J, Morais J, et al. Reversal strategies for nonvitamin $\mathrm{K}$ antagonist oral anticoagulants: a critical appraisal of available evidence and recommendations for clinical management-a joint position paper of the European Society of Cardiology Working Group on Cardiovascular Pharmacotherapy and European Society of Cardiology Working Group on Thrombosis. Eur Heart J. 2017;38(22):1710-1716. 
18. Salzano A, Proietti M, D’Assante R, Saldamarco L, Cittadini A, Paladino F. Bleeding related to non-vitamin $\mathrm{K}$ antagonist oral anticoagulants in emergency department: A "Real-world" snapshot from Southern Italy. On behalf of MIRC-NOAC study group. Eur J Intern Med. 2018;48:e21-e24.

19. Tomaselli GF, Mahaffey KW, Cuker A, et al. 2017 ACC Expert Consensus Decision Pathway on Management of Bleeding in Patients on Oral Anticoagulants. J Am Coll Cardiol. 2017;70(24):3042-3067.
20. Verma A, Ha ACT, Rutka JT, Verma S. What surgeons should know about non-vitamin K oral anticoagulants: A review. JAMA Surg. 2018; 153(6):577.

21. Levy JH, Douketis J, Weitz JI. Reversal agents for non-vitamin K antagonist oral anticoagulants. Nat Rev Cardiol. 2018;15(5):273-281.

\section{Publish your work in this journal}

Therapeutics and Clinical Risk Management is an international, peerreviewed journal of clinical therapeutics and risk management, focusing on concise rapid reporting of clinical studies in all therapeutic areas, outcomes, safety, and programs for the effective, safe, and sustained use of medicines. This journal is indexed on PubMed Central, CAS,
EMBase, Scopus and the Elsevier Bibliographic databases. The manuscript management system is completely online and includes a very quick and fair peer-review system, which is all easy to use. Visit http://www.dovepress.com/testimonials.php to read real quotes from published authors.

Submit your manuscript here: http://www.dovepress.com/therapeutics-and-clinical-risk-management-journal 